\title{
CLOT FORMATION AND LYSIS IN PLATELET RICH PLASMA OF HEALTHY DONORS AND PATIENTS WITH RESISTANT HYPERTENSION
}

\author{
I. I. PATALAKH ${ }^{1}$, O. V. REVKA ${ }^{1 凶}$, O. B. KUCHMENKO', \\ O. O. MATOVA', T. F. DROBOTKO ${ }^{2}$, T. V. GRINENKO \\ ${ }^{1}$ Palladin Institute of Biochemistry, National Academy of Sciences of Ukraine, Kyiv; \\ e-mail: sedrickedel@gmail.com \\ ${ }^{2}$ National Scientific Center "Strazhesko Institute of Cardiology” NAMS of Ukraine, Kyiv
}

Hemostatic balance in blood is affected by numerous factors, including coagulation and fibrinolytic proteins, the wide spectrum of their inhibitors, and blood cells. Since platelets can participate in contradictory processes, they significantly complicate the whole picture. Therefore, nowadays the development of global assays of hemostasis, which can reflect the physiological process of hemostasis and can be used for point-of-care diagnosis of thrombosis, is crucial. This paper outlines a new approach we used to analyze the capabilities of clot waveform analysis tools to distinguish the response of platelet-rich plasma from healthy donors and patients with arterial hypertension caused by stimulation of coagulation and lysis (with exogenous thrombin and recombinant tissue-type plasminogen activator, respectively). In donor plasma, when the clot degradation was accompanied by $40 \mathrm{IU} / \mathrm{ml}$ of recombinant tissue-type plasminogen activator, platelets potentiated fibrinolysis more than coagulation, which ultimately shifts the overall balance to a profibrinolytic state. At the same time, for patients with hypertension, platelets, embedded in clot obtained from platelet-rich plasma, showed a weaker ability to stimulate fibrinolysis. The obtained data gives the evidence that platelets can act not only as procoagulants but also as profibrinolytics. By simultaneously amplifying coagulation and fibrinolysis, making their rates comparable, platelets would control plasma procoagulant activity, thereby regulating local hemostatic balance, the size and lifetime of the clot. Moreover, clot waveform analysis may be used to distinguish the effects of platelet-rich plasma on clotting or lysis of fibrin clots in healthy donors and patients with essential hypertension.

Ke y wo rd s : platelets, clotting, lysis, clot waveform analysis, arterial hypertension.

$\mathrm{B}$ lood coagulability, the size and stability of the formed clot, and its lifetime and degradation due to fibrinolysis are considered as factors responsible for the repair of damaged endothelium and the restoration of blood circulation. Stimulated vascular endothelial cells in response to various stimuli can release into circulation a wide spectrum of coagulative and fibrinolytic proteins and promote platelet activation. Stimulus-dependent increase of factor VIII clotting activity, von Willebrand factor antigen, and tissue-type plasminogen activator (t-PA) are found after intravenous administration of epinephrine [1]; increased plasma levels of plasminogen activator inhibitor type 1 (PAI-1), $\mathrm{t}-\mathrm{PA}$, fibrinogen, and thrombomodulin are associa- ted with a procoagulant tendency and endothelial damage [2, 3]. The prognostic value of alpha 2-antiplasmin and thrombin activatable fibrinolysis inhibitor (TAFI) are useful for myocardial infarction, but other fibrinolytic components are not significant as cardiovascular risk factors [3]. Activated platelets can complicate the picture. As the main source of PAI-1 in blood, they can inhibit and simultaneously, as an alternative surface cofactor, ensure the activation of plasminogen by t-PA within the plasmingenerating pathway [4]. Consequently, dynamic changes between activators and inhibitors, produced by platelets, may considerably change the overall hemostatic balance. It is obvious that the assessment of such numerous changes in circulation, which may

(C) 2018 Patalakh I. I. et al. This is an open-access article distributed under the terms of the Creative Commons Attribution License, which permits unrestricted use, distribution, and reproduction in any medium, provided the original author and source are credited. 
reinforce or compensate each other, requires new integrated approaches. Thus, some global assays are currently being proposed that aim to mimic and reflect the major physiological aspects of the hemostasis process in vitro [5-7], especially global assays that simultaneously measures both coagulation and fibrinolysis [8-10].

Global analyses can be a good alternative to traditional coagulation tests, such as prothrombin time (PT) or activated partial thromboplastin time (APTT), which are insensitive to hypercoagulation and unable to assess inconsistency between coagulation and fibrinolysis in certain pathological states with platelet and endothelial anomalies, such as essential hypertension. Patients with hypertension have impaired fibrinolytic activity due to increased PAI-1 activity and antigen level, as well as a significant decrease in t-PA activity [11-13].

The complex interactions between these fibrinolytic markers and their unpredictable overall effect on coagulation and fibrinolysis can make them less suitable for controlling the development of hypertension.

In our work we studied the ability of plateletrich plasma (PRP) after stimulation of coagulation and fibrinolytic systems to form a fibrin clot with incorporated platelets; we also assessed the integral effect of platelets on the evolution of the fibrin clot. For these purposes, we used our modification of clot waveform analysis - one of the global coagulation assays, principally based on the traditional APTT assay, which makes possible the evaluation of the dynamics of clot formation and lysis in real time [14]. In addition, we analyzed the capabilities of waveform analysis tools to distinguish the PRP response of healthy donors and patients with essential hypertension caused by stimulation of coagulation and lysis (with exogenous thrombin and recombinant $\mathrm{t}-\mathrm{PA}$, respectively).

\section{Materials and Methods}

Blood obtained by venipuncture was added to acid citrate dextrose (ACD) solution $(85 \mathrm{mmol} / \mathrm{l}$ trisodium citrate, $65 \mathrm{mmol} / \mathrm{l}$ citric acid, and $100 \mathrm{mmol} / \mathrm{l} \mathrm{D}$-glucose), in the proportions of $6 \mathrm{vol}$ of blood +1 vol of ACD. All donors were informed of the use of their blood samples for the purposes of the present study.

The study included plasma samples from 16 patients with resistant arterial hypertension (mean age $51.4 \pm 2.6$ years) undergoing treatment at the
National Scientific Center "Strazhesko Institute of Cardiology" NAMS of Ukraine. The duration of the disease was an average of $13.4 \pm 2.0$ years. As a control group we enrolled 7 healthy persons (mean age $49.2 \pm 2.1$ years). The diagnosis of resistant arterial hypertension was carried out in accordance with the recommendations of the European Society of Hypertension and the European Society of Cardiology. In particular, blood pressure at the doctor's appointment was more than $140 / 90 \mathrm{~mm} \mathrm{Hg}$ with the inclusion of patients with three or more antihypertensive drugs at optimal doses, if one of them is a diuretic.

The exclusion criteria were treatment with nonsteroidal anti-inflammatory drugs, sympathomimetics (some drugs for weight loss, decongestants), stimulants, oral contraceptives, and some medicinal plants (Glycyrrhiza).

After ACD-blood was gently centrifuged at $180 \mathrm{~g}$ during $20 \mathrm{~min}$, supernatant was taken and centrifuged at $1000 \mathrm{~g}$ during $15 \mathrm{~min}$. Second supernatant, platelet-pure plasma (PPP), was decanted and the remaining $1 \mathrm{ml}$ of platelet suspension was used for measurement of platelet count. PPP was centrifuged at $12000 \mathrm{~g}, 7 \mathrm{~min}$ for platelet-free plasma (PFP) preparation. PRP with platelet count $300 \times 10^{6} / \mathrm{ml}$ was obtained by diluting the platelet suspension with an appropriate volume of PFP.

Testing of platelet function was carried out within $3 \mathrm{~h}$ after collection of blood. Before testing PRP aggregability to thrombin (1 NIH units/ml) was verified, platelets that showed a weakened response to thrombin or exhibited spontaneous aggregation were excluded from the experiment.

Procoagulant activity of platelets was assessed without the use of non-physiological test reagents (ellagic acid, caoline) by a one-stage clotting test [15]. In fact, clotting was initiated by restoring the plasma level of $\mathrm{Ca}^{2+}$ (recalcified plasma): $0.05 \mathrm{ml}$ of PRP as a cell sample, $0.05 \mathrm{ml}$ of Tris- $\mathrm{HCl}$ buffer $(0.05 \mathrm{M}$, $\mathrm{pH} 7.4$ ), and $0.05 \mathrm{ml}$ of $0.025 \mathrm{M} \mathrm{CaCl}_{2}(8 \mathrm{mM}$, final). As a control, the autologous fresh PFP was clotted instead of PRP in the same system. In parallel experiments, the coagulation of PRP and PFP was initiated with a mixture of $0.025 \mathrm{M} \mathrm{CaCl}_{2}$ and $0.5 \mathrm{nM}$ thrombin with a final concentrations $8 \mathrm{mM}$ and $0.17 \mathrm{nM}$, respectively.

The lysis was stimulated by the addition of recombinant t-PA (rt-PA) (15 and $40 \mathrm{IU} / \mathrm{ml}$, final) to the clotting system at the same time as the coagulation initiator(s), so that the polymerization occurred following lysis. The concentration of rt-PA 
was adjusted according to an assumed average level of PAI-1 in PFP and PRP, which provided complete fibrinolysis for not more than $3 \mathrm{~h}$.

Procedures for the preparation and testing of platelets were made without contact with glass or other negatively charged materials to minimize the activation of factor XII. To stimulate the fibrinolytic system by activating endogenous plasminogen, rt-PA (as an exogenous t-PA) in the volume of Tris- $\mathrm{HCl}$ buffer was mixed with plasma just before clotting.

The coagulation process as well as the interrelated coagulation and fibrinolysis in the test samples were evaluated by changes in the optical density (absorbance) of the plasma (in OD units) that were monitored at $405 \mathrm{~nm}$ every $5 \mathrm{~min}$ in a Multiscan 2000 microplate reader (Thermo Electron Corporation, Vantaa, Finland). Fibrinolysis was measured by recording the decrease in OD during dissolution of plasma clots containing platelets, or control cell-free plasma clots. PPP and PRP had different effects on baseline absorption, therefore such absorption was subtracted as a blank and does not affect assay data.

Clot formation was observed until a constant maximum absorption in two parallel samples was achieved. In the internal lysis model, measurements were continued until a complete lysis of the clots in the presence of platelets was detected (or no more than $3 \mathrm{~h}$ ). By plotting the OD units against time, the OD ( $\mathrm{t}$ ) curve was obtained and some kinetic parameters were calculated.

The following parameters were chosen as the most informative to distinguish the group of patients:

- The maximum absorbance (OD ${ }_{\max }$, OD units) gives information on the final point (peak) of fibrin polymerization.

- The time to peak (min) was calculated as the time from the start of coagulation (zero point) to the steepest curve turning to peak.

- The clot lifetime ( $\mathrm{min}$ ) was defined as the time required to return the OD to the baseline level from the onset of clotting of the plasma to the time of complete dissolution of the formed clot.

- The area under the curve (AUC, OD units-min) was used to evaluate the local hemostatic balance (LHB) in plasma, including the balance between the procoagulant and the profibrinolytic action of the platelets. The augmentation of plateletdependent AUC values in comparison with PFP-derived clot was estimated as an LHB shift to a more procoagulant state. Alternatively, AUC value reduction was considered as the more profibrinolytic state of LHB.
Statistical analyses. The effect of platelets on clotting and clot lysis was analyzed by comparing the corresponding parameters for autologous PFP and PRP of donors using the paired Student's $t$-test. Data are shown in Table 1 as means and standard error of mean (SEM). $P$ values of less than 0.05 were considered as statistically significant. Differences between unpaired groups of donors and patients were analyzed by the Mann-Whitney-U test at the chosen $\alpha$-level (0.05 or 0.01). Data are shown in Table 2 as median and median absolute deviation (MAD). All data were calculated using the Microsoft Excel software (Microsoft Corporation, Redmond, Washington, U.S.).

\section{Results and Discussion}

To distinguish the effects of platelets and plasma proteins on coagulation parameters, the clotting of autologous PFP and PRP was compared after reconstitution of the physiological level of calcium. Under these conditions, the procoagulant action of platelets on the polymerization of fibrin was confirmed by shortening the lag time from $34.3 \pm 9.8 \mathrm{~min}$ to $10.0 \pm 2.9 \mathrm{~min}$, for PFP and PRP, respectively (Fig. 1).

As the recalcification procedure restores the activity of coagulation factors II, VII, IX, X, this result elucidates the ability of platelets to stimulate thrombin production through the intrinsic pathway of coagulation.

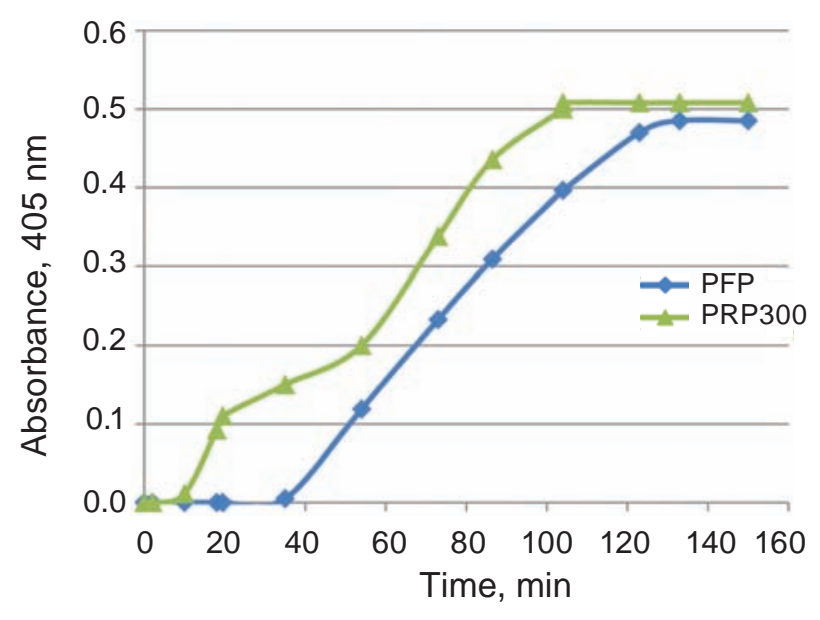

Fig. 1. Clot waveform absorbance pattern which reflects the kinetics of clotting in control plasma of healthy donors after recalcification: $P F P$ - plateletfree plasma, PRP300 - platelet rich plasma with $300 \times 10^{6}$ platelets $/ \mathrm{ml}$ 
A small amount of exogenous thrombin then was combined with $\mathrm{CaCl}_{2}$ to check the contribution of platelets to the regulation of coagulation through a positive feedback loop catalyzed by thrombin. As a result, in this model system lag-phase was canceled, coagulation progressed faster and was reflected by the maximum absorbance of $0.508 \pm 0.035$ for PFP (Fig. 2, A) and $0.454 \pm 0.031$ for PRP (Fig. 2, B). The time to peak during PRP clotting was shortened by an average of 15.7 min compared to PFP, which confirmed the stimulating effect of platelets on clotting independent of exogenous thrombin.

For most of the PRP samples tested (71.4\%), the increase in OD to a maximum was accompanied by its subsequent decrease, which indicates the conversion of the polymerization of fibrin into its spontaneous lysis. The LHB between these opposing processes was estimated by the AUC, which was calculated after processing the results of the clot waveform analysis. By comparing AUC for PFP and PRP (65.2 \pm 2.2 and $31.4 \pm 4.1$, respectively) (Ta-

$\boldsymbol{A}$

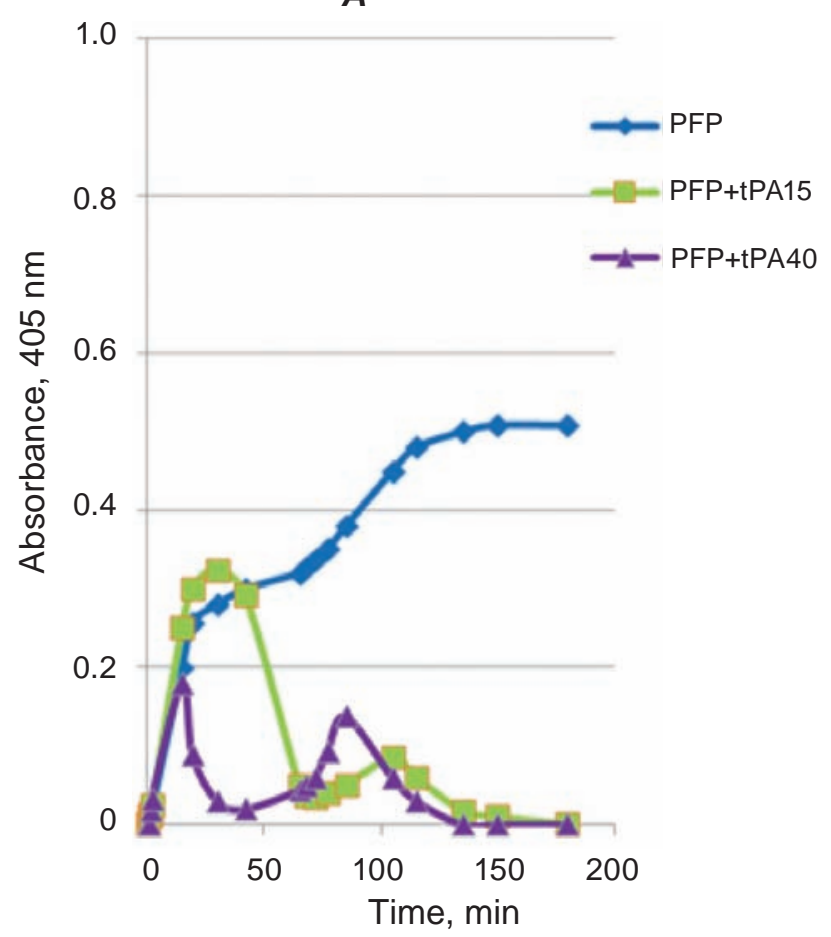

ble 1), the ratio $\mathrm{AUC}_{\mathrm{PRP}} / \mathrm{AUC}_{\mathrm{PFP}}=0.48$ was calculated; this indicates a strong profibrinolytic action of platelets in the LHB in control plasma.

The next step was to determine the effect of platelets on the polymerization of fibrin succeeded by fibrinolysis. Indeed, rt-PA in concentrations of 15 and $40 \mathrm{IU} / \mathrm{ml}$ dramatically reduced the time to peak for both the cell-free clots and platelet-containing clots (Table 1) indicating that fibrinolysis begins to counteract the coagulation process in the early stages of fibrin polymerization. The maximum absorbance was significantly reduced during clotting of PRP and PFP in the presence of 15 and 40 IU/ $\mathrm{ml}$ rt-PA compared with clotting without exogenous t-PA (Table 1), which additionally confirms the early onset of the concurrent fibrinolysis.

Under these conditions, a biphasic waveform for the change in PFP absorption was obtained for the clotting/lysis process (Fig. 1, A), while platelets in the PRP contributed to a decrease in the second phase (Fig. 1, B).

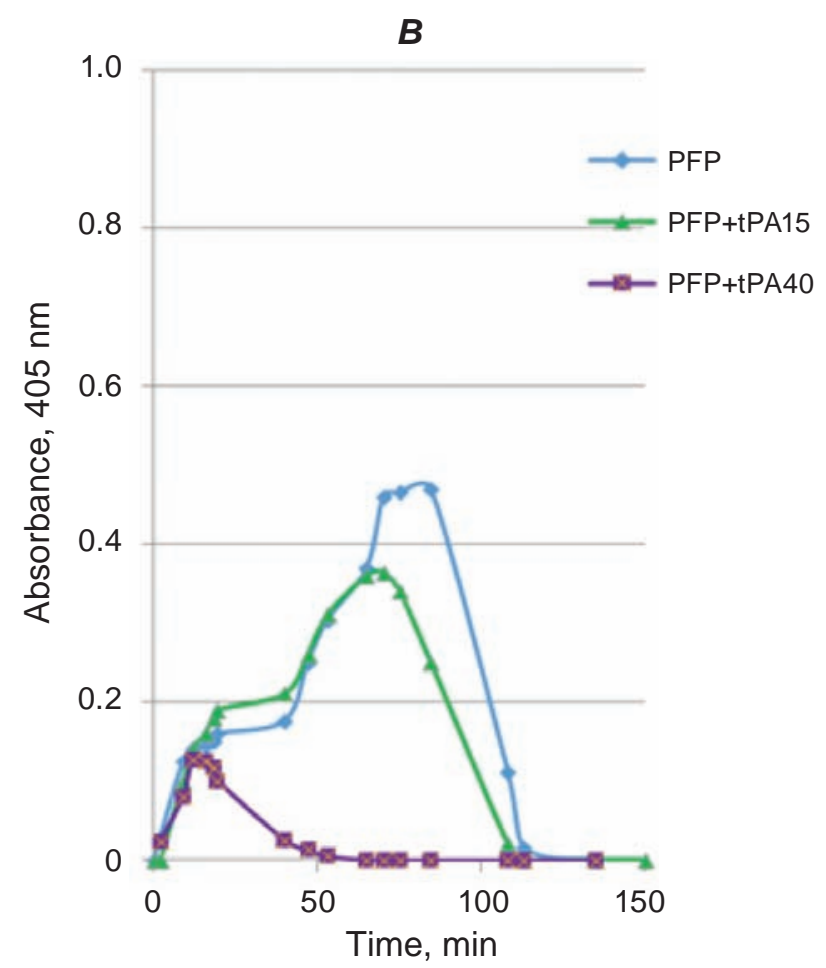

Fig. 2. Clot waveform absorbance pattern which reflects the kinetics of clotting and clot lysis in control plasma of healthy donors. (A) PFP - clotting of recalcified PFP with thrombin, $0.17 \mathrm{nM}$; PFP + tPA15 - clotting with subsequent lysis, stimulated by rt-PA, $15 \mathrm{IU} / \mathrm{ml}$; PFP + tPA40 - clotting with subsequent lysis, stimulated by rt-PA, $40 \mathrm{IU} / \mathrm{ml}$; (B) PRP - clotting of recalcified PRP with thrombin, $0.17 \mathrm{nM}$; $P R P+t P A 15$ - clotting with subsequent lysis, stimulated by rt-PA, $15 \mathrm{IU} / \mathrm{ml}$; PRP+tPA40 - clotting with subsequent lysis, stimulated by rt-PA, $40 \mathrm{IU} / \mathrm{ml}$. PFP - platelet-free plasma, PRP - platelet-rich plasma, rt-PA - recombinant tissue-type plasminogen activator 
Table 1. Clot waveform analysis (CWA) parameters for control PFP- and PRP-derived clots (all values are presented as mean \pm SEM)

\begin{tabular}{|c|c|c|}
\hline Parameter & PFP & PRP \\
\hline \multicolumn{3}{|c|}{ Clotting } \\
\hline $\mathrm{OD}_{\max }$ & $0.503 \pm 0.037$ & $0.454 \pm 0.031$ \\
\hline Time to peak & $100.0 \pm 9.2$ & $84.3 \pm 6.4$ \\
\hline AUC & $65.2 \pm 4.0$ & $\begin{array}{c}31.4 \pm 4.1^{*} \\
(\mathrm{p}=0.0002)\end{array}$ \\
\hline \multicolumn{3}{|c|}{ Clotting/lysis, $15 \mathrm{IU} / \mathrm{ml} \mathrm{rt}-\mathrm{PA}$} \\
\hline $\mathrm{OD}_{\max }$ & $0.23 \pm 0.025$ & $0.364 \pm 0.088$ \\
\hline Time to peak & $21.7 \pm 1.7$ & $66.3 \pm 16.8$ \\
\hline AUC & $15.7 \pm 2.5$ & $18.5 \pm 3.5$ \\
\hline Clot lifetime & $146.7 \pm 12.0$ & $143.8 \pm 42.7$ \\
\hline \multicolumn{3}{|c|}{ Clotting/lysis, $40 \mathrm{IU} / \mathrm{ml}$ rt-PA } \\
\hline $\mathrm{OD}_{\max }$ & $0.180 \pm 0.031$ & $0.144 \pm 0.021$ \\
\hline Time to peak & $13.6 \pm 0.9$ & $11.7 \pm 2.5$ \\
\hline AUC & $9.5 \pm 2.9$ & $4.0 \pm 0.6$ \\
\hline Clot lifetime & $134.2 \pm 20.8$ & $\begin{array}{c}56.4 \pm 10.8^{*} \\
(p=0.011)\end{array}$ \\
\hline
\end{tabular}

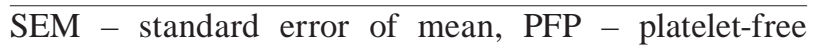
plasma, PRP - platelet-rich plasma, OD - optical density, AUC - area under the curve, rt-PA - recombinant tissuetype plasminogen activator. *Means differ significantly between PFP and PRP groups as confirmed by the paired Student's $t$-test with the indicated $p$-value.

It is important to note, that platelets affect the clotting/lysis parameters differently in the presence of 15 and $40 \mathrm{IU} / \mathrm{ml}$ rt-PA. When $15 \mathrm{IU} / \mathrm{ml}$ of rt-PA was used, there was a tendency to increase $\mathrm{OD}_{\max }$ in the presence of cells within the clot $(0.364 \pm 0.088$ versus $0.23 \pm 0.025$ for PFP- and PRP-derived clots, respectively). Despite the fact that the platelets prolonged the time to peak, they nevertheless provided an increase in AUC of the PRP-derived clot as compared to the cell-free clot $(18.5 \pm 3.5$ vs. $15.7 \pm 2.5$, respectively) (Table 1). Accordingly, in the presence of $15 \mathrm{IU} / \mathrm{ml}$ rt-PA, the AUCPRP/AUCPFP ratio was 1.2 , indicating a procoagulant effect of platelets. In contrast, the clot lifetime shortened 1.5 times (from $134.2 \pm 20.8$ to $56.4 \pm 10.8$ ), and the AUC decreased more than 2 times (from $9.5 \pm 2.9$ to $4.0 \pm 0.6$ ) for the platelet-containing clot in comparison to the clot obtained from PFP if $40 \mathrm{IU} / \mathrm{ml}$ rt-PA was added (Table 1). The ratio $\mathrm{AUC}_{\mathrm{PRP}} / \mathrm{AUC}_{\mathrm{PFP}}=0.42$ indicates the maximum manifestation of the profibrinolytic properties of platelets under these conditions.

The control parameters of clotted PFP and PRP from donors were compared with similar parameters, which were evaluated for patients with arterial hypertension. A statistically significant increase in almost all plasma clotting parameters (for both PFP and PRP) in patients compared with donors (Table 2) indicates the development of hypercoagulable symptoms in hypertension. Stimulation of fibrinolysis by exogenous rt-PA in both concentrations did not significantly change this trend. For PFP-derived clots ODmax remained higher in comparison with the control by 1.7 times and 1.8 times after the addition of rt-PA $(0.436 \pm 0.131$ and $0.301 \pm 0.040$ for PFP of patients vs. $0.253 \pm 0.044$ and $0.170 \pm 0.025$ for PFP of donors with 15 and $40 \mathrm{IU} / \mathrm{ml}$ rt-PA, respectively) (Table 2). When stimulated with $15 \mathrm{IU} /$ $\mathrm{ml}$ rt-PA, none of the clotted PFP was dissolved by at least $50 \%$ in $3 \mathrm{~h}$. Even a strong stimulation of $40 \mathrm{IU} /$ $\mathrm{ml}$ rt-PA did not return the clot lifetime to the control value; it remained on average longer for $45 \mathrm{~min}$ compared to clots from the control PFP (185.0 \pm 25.0 vs. $140.0 \pm 60.0$ for the patient's and control PFP, respectively).

In PRP of patients, platelets had increased $\mathrm{OD}_{\text {max }}$ relative to PFP regardless of the addition of rt-PA (Table 2) and did not affect the second phase of the clotting/lysis process. At the same time, they promoted weak spontaneous lysis and lysis stimulated by $15 \mathrm{IU} / \mathrm{ml}$ rt-PA. Profibrinolytic properties of platelets from PRP of patients were inconsistent: only in $55.6 \%$ of patients the coagulated PRP was then able to lyse spontaneously, but such a degradation of the clot was never completed during monitoring time.

However, the platelets, incorporated in the PRP-derived clot, reduced its AUC relative to the PFP-derived clot (124.5 \pm 40.2 and $143.8 \pm 31.9$, respectively) (Table 2). Accordingly, the obtained AUCPRP/AUCPFP ratio $=0.86$ indicates a certain profibrinolytic shift of the LHB in PRP of patients. In contrast to donors, the LHB in plasma of patients supplemented with $40 \mathrm{IU} / \mathrm{ml}$ rt-PA was shifted to the procoagulant state, which is confirmed by the ratio $\mathrm{AUC}_{\mathrm{PRP}} / \mathrm{AUC}_{\mathrm{PFP}}=1.2\left(\mathrm{AUC}_{\mathrm{PRP}}=25.1 \pm 2.5\right.$ and $\mathrm{AUC}_{\mathrm{PFP}}=21.0 \pm 8.0$ from Table 2).

Changes in coagulation/lysis parameters in PRP supplemented with $40 \mathrm{IU} / \mathrm{ml}$ rt-PA were the most significant between donors and patients, which 
Ta ble 2. Clot waveform analysis (CWA) parameters for donor and patient plasma samples (all values are presented as medians $\pm M A D$ )

\begin{tabular}{|c|c|c|c|}
\hline Parameter & Donors $(n=7)$ & Patients $(n=16)$ & $\alpha$-level \\
\hline \multicolumn{4}{|c|}{ Platelet-free plasma } \\
\hline \multicolumn{4}{|c|}{ Clotting } \\
\hline $\mathrm{OD}_{\max }$ & $0.486 \pm 0.051$ & $0.582 \pm 0.157$ & \\
\hline Time to peak & $105.0 \pm 15.0$ & $145.0 \pm 30.0^{*}$ & $\alpha=0.01$ \\
\hline AUC & $68.4 \pm 3.9$ & $143.8 \pm 31.9 *$ & $\alpha=0.01$ \\
\hline \multicolumn{4}{|c|}{ Clotting/lysis, $15 \mathrm{IU} / \mathrm{ml} \mathrm{rt}-\mathrm{PA}$} \\
\hline $\mathrm{OD}_{\max }$ & $0.253 \pm 0.044$ & $0.436 \pm 0.131$ & \\
\hline Time to peak & $22.5 \pm 2.5$ & $115.0 \pm 35.0^{*}$ & $\alpha=0.05$ \\
\hline AUC & $18.2 \pm 5.3$ & $83.7 \pm 22.5^{*}$ & $\alpha=0.05$ \\
\hline Clot lifetime & $155.0 \pm 20.0$ & $>300 \mathrm{~min}$ & \\
\hline \multicolumn{4}{|c|}{ Clotting/lysis, 40 IU/ml rt-PA } \\
\hline $\mathrm{OD}_{\max }$ & $0.170 \pm 0.025$ & $0.301 \pm 0.040^{*}$ & $\alpha=0.01$ \\
\hline Time to peak & $15.0 \pm 0.0$ & $35.0 \pm 15.0^{*}$ & $\alpha=0.01$ \\
\hline AUC & $9.1 \pm 4.2$ & $21.0 \pm 8.0^{*}$ & $\alpha=0.01$ \\
\hline Clot lifetime & $140.0 \pm 60.0$ & $185.0 \pm 25.0 *$ & $\alpha=0.05$ \\
\hline \multicolumn{4}{|c|}{ Platelet-rich plasma } \\
\hline \multicolumn{4}{|c|}{ Clotting } \\
\hline $\mathrm{OD}_{\max }$ & $0.418 \pm 0.064$ & $0.691 \pm 0.129 *$ & $\alpha=0.01$ \\
\hline Time to peak & $80.0 \pm 10.0$ & $123.7 \pm 26.3^{*}$ & $\alpha=0.01$ \\
\hline AUC & $32.4 \pm 10.1$ & $124.5 \pm 40.2^{*}$ & $\alpha=0.01$ \\
\hline \multicolumn{4}{|c|}{ Clotting/lysis, $15 \mathrm{IU} / \mathrm{ml}$ rt-PA } \\
\hline $\mathrm{OD}_{\max }$ & $0.365 \pm 0.172$ & $0.533 \pm 0.153$ & \\
\hline Time to peak & $72.5 \pm 15.0$ & $95.0 \pm 32.5$ & \\
\hline AUC & $24.3 \pm 10.1$ & $52.3 \pm 29.5$ & \\
\hline Clot lifetime & $110.0 \pm 17.5$ & $130.0 \pm 35.0$ & \\
\hline \multicolumn{4}{|c|}{ Clotting/lysis, $40 \mathrm{IU} / \mathrm{ml}$ rt-PA } \\
\hline $\mathrm{OD}_{\max }$ & $0.116 \pm 0.007$ & $0.315 \pm 0.118^{*}$ & $\alpha=0.01$ \\
\hline Time to peak & $10.0 \pm 3.0$ & $40.0 \pm 10.0^{*}$ & $\alpha=0.01$ \\
\hline AUC & $4.3 \pm 2.1$ & $25.1 \pm 2.5^{*}$ & $\alpha=0.01$ \\
\hline Clot lifetime & $50.0 \pm 20.0$ & $140.0 \pm 20.0^{*}$ & $\alpha=0.01$ \\
\hline
\end{tabular}

MAD - median absolute deviation, OD - optical density, AUC - area under the curve; rt-PA - recombinant tissuetype plasminogen activator. *Medians differ significantly between donors and patients as confirmed by U-test statistics (Uobt $\leq$ Ucrit at the chosen $\alpha$-level).

is confirmed by statistical validity (Table 2). These parameters and these testing conditions can be most indicative for individualized monitoring of the hemostatic system of patients with resistant hypertension.
It should be noted that there are significant individual differences in some of the estimated parameters (Fig. 3), especially, inside the patient group. Wide variability of PRP responses caused by stimulation of coagulation and lysis can correlate with the 

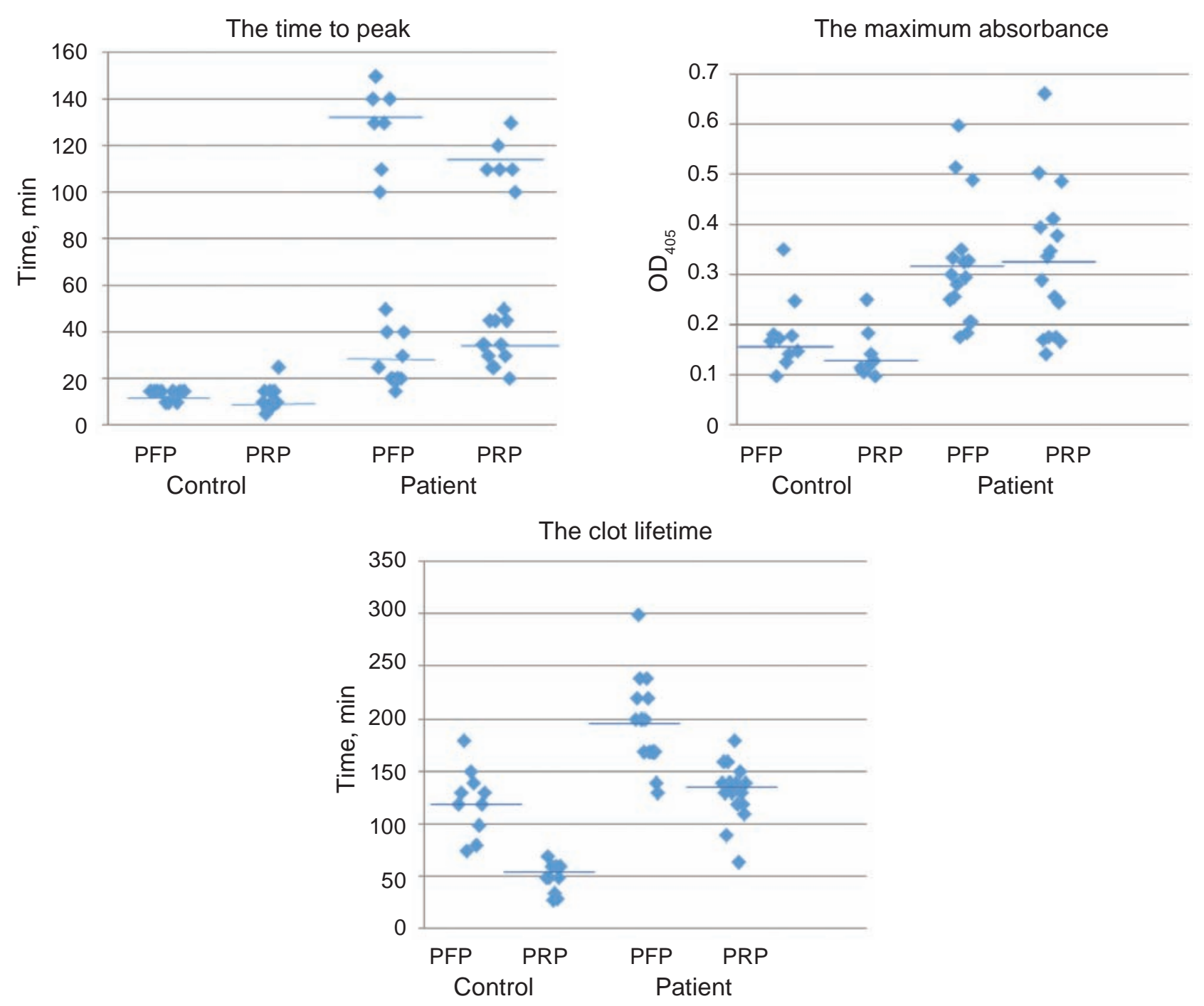

Fig. 3. Individual parameters of the clotting/lysis process in PFP and PRP of donors (control) and patients with arterial hypertension, stimulated by $0.17 \mathrm{nM}$ of thrombin and $40 \mathrm{IU} / \mathrm{ml}$ of $\mathrm{rt}-\mathrm{PA}$. Horizontal markers indicate the average value of the parameter. PFP - platelet-free plasma, PRP-platelet-rich plasma, OD-optical density, rt-PA - recombinant tissue-type plasminogen activator

degree of disease manifestation and can be useful for a personalized prognosis of the course of the disease.

A simple global assay, based on the clot waveform analysis, which was used in our study, allowed us to estimate the cumulative effect of platelets on the clotting process and subsequent fibrinolysis. The data obtained are somewhat inconsistent with the traditional view of platelets as exclusively procoagulant cells responsible for the propagation of thrombin generation and polymerization of fibrin. Factually, the acceleration of the time to peak (Table 1) indicates the manifestation of procoagulant properties of platelets; but their profibrinolytic effect was more pronounced, which is confirmed by a decrease in the AUC value for clotted PRP as compared with PFP.
Thus, the ratio $\mathrm{AUC}_{\mathrm{PRP}} / \mathrm{AUC}_{\mathrm{PFP}}=0.48$ indicates that thrombin-stimulated platelets potentiate fibrinolysis more than coagulation, thereby shifting the overall balance towards fibrinolysis.

After the addition of $15 \mathrm{IU} / \mathrm{ml}$ rt-PA in PFP and PRP, the differences in the coagulation and lysis parameters for them were insignificant (Table 1). Apparently, activated platelets enhance coagulation by additional generation of thrombin and, at the same time, reduce fibrinolysis by secretion of PAI-1 into clotting plasma. The ratio $\mathrm{AUC}_{\mathrm{PRP}} / \mathrm{AUC}_{\mathrm{PFP}}=1.18$ confirm that platelets in plasma supplemented with a small amount of exogenous rt-PA act rather as procoagulants. When the clot degradation was accompanied by $40 \mathrm{IU} / \mathrm{ml} \mathrm{rt}-\mathrm{PA}$, added to overlap platelet- 
secreted PAI-1, the platelets normally restored their profibrinolytic properties.

For patients with hypertension, platelets, embedded in the PRP-derived clot, showed a weak ability to stimulate fibrinolysis. For example, spontaneous lysis of this clot causes only a $22 \%$ decrease in AUC compared to the clot obtained from PFP, whereas the difference for control clotted PRP was 48.1\%.

PRP and PFP in patients were also significantly less sensitive to rt-PA. It looks like only a certain constant amount of total t-PA provides lysis in accordance with a certain constant platelet count; the rest of the exogenous activator does not work properly in patients' PRP. The observation that platelets can protect plasmin and t-PA from inhibition by localization on activated surfaces [4] can help explain this fact.

The present findings of decreased stimulatory effect of platelets on the rt-PA-dependent lysis of clotted plasma in patients compared with donors are supported by work of the groups of Poli and colleagues [16] where they found a decreased fibrinolytic state in hypertension due to elevated levels of both tPA antigen and PAI-1 antigen. It has been shown that most of the t-PA antigen is complexed with PAI-1 and is inactive [17]. This can explain why the exogenous rt-PA in our study, added to plasma in an increased amount, does not help to adequately accelerate fibrinolysis. Obviously, this amount of rtPA was lower than the PAI-1 level in the patients' plasma.

In our study, we confirmed the procoagulant effect of platelets on fresh donor plasma, where the endogenous t-PA concentration is inadequate to exceed the inhibitory effect of total PAI-1 derived from plasma and platelets, so fibrinolysis stimulation is slow and has little effect on clotting. On the other hand, we have shown that during clot formation and lysis, platelets can effectively promote fibrinolysis, despite PAI-1, which they secrete, if the amount of t-PA in the medium is sufficient to neutralize the inhibitor. This may be important for the development of a blood clot in the bloodstream, since in blood vessels, the plasma t-PA level can increase several folds due to local activation of the endothelium.

Our study is limited to a small number of patients; nevertheless, it demonstrates the usefulness of clot waveform analysis for evaluating the net effects of different platelet activities. We propose to test PRP instead of PFP, traditionally used in conven- tional coagulation assays that will allow monitoring the balance between procoagulant and anticoagulant events in a plasma sample. We believe that this approach can provide a more reliable differentiation of healthy subjects and patients, and also improve therapeutic approaches for treating the disease.

\section{Ethical Approval}

All participants (donors and patients) gave written informed consent and the studies were approved by the local research ethics committees of Palladin Institute of Biochemistry of the National Academy of Sciences of Ukraine (No 3, from 30.08.2017).

\section{Acknowledgements}

Authors thank Cedars - Sinai Medical Center's International Research and Innovation in Medicine Program, the Association for Regional Cooperation in the Fields of Health, Science and Technology (RECOOP HST Association) for their support of our organization as participating Cedars - Sinai Medical Center - RECOOP Research Centers (CRRC).

\section{Financial support}

This work was supported by National Academy of Sciences of Ukraine, theme No 2.2.10, No 15 in 2017.

\section{References}

1. von Känel R, Dimsdale JE. Effects of sympathetic activation by adrenergic infusions on hemostasis in vivo. Eur J Haematol. 2000; 65(6): 357-369.

2. Hrafnkelsdottir T, Gudnason T, Wall U, Jern C, Jern S. Regulation of local availability of active tissue-type plasminogen activator in vivo in man. J Thromb Haemost. 2004; 2(11): 19601968.

3. Chapin JC, Hajjar KA. Fibrinolysis and the control of blood coagulation. Blood Rev. 2015; 29(1): 17-24.

4. Michelson AD. Platelets. Academic Press, 2013; 3 ed. $1398 \mathrm{p}$.

5. Lipets EN, Ataullakhanov FI. Global assays of hemostasis in the diagnostics of hypercoagulation and evaluation of thrombosis risk. Thromb $J$. 2015; 13(1): 4.

6. Lancé MD. A general review of major global coagulation assays: thrombelastography, thrombin generation test and clot waveform analysis. Thromb J. 2015; 13: 1.

7. Kremers RM, Peters TC, Wagenvoord RJ, Hemker HC. The balance of pro- and 
anticoagulant processes underlying thrombin generation. J Thromb Haemost. 2015; 13(3): 437447.

8. Tripodi A. A(nother) test meant to fill the gap between in vivo and ex vivo hemostasis. Clin Chem. 2014; 60(9): 1137-1140.

9. van Geffen M, Loof A, Lap P, Boezeman J, Larosvan Gorkom BA, Brons P, Verbruggen B, van Kraaij M, van Heerde WL. A novel hemostasis assay for the simultaneous measurement of coagulation and fibrinolysis. Hematology. 2011; 16(6): 327-336.

10. Antovic A. The overall hemostasis potential: a laboratory tool for the investigation of global hemostasis. Semin Thromb Hemost. 2010;3 6(7): 772-779.

11. Tabak O, Gelisgen R, Uzun H, Kalender B, Balci H, Curgunlu A, Simsek G, Karter Y. Hypertension and hemostatic/fibrinolytic balance disorders. Clin Invest Med. 2009; 32(6): E285.

12. Lip GY, Blann AD. Endothelium and fibrinolysis in hypertension: important facets of a prothrombotic state? Hypertension. 2008; 52(2): 218-219.
13. Jeng JR, Sheu WH, Jeng CY, Huang SH, Shieh SM. Impaired fibrinolysis and insulin resistance in patients with hypertension. Am J Hypertens. 1996; 9(5): 484-490.

14. Sevenet PO, Depasse F. Clot waveform analysis: Where do we stand in 2017? Int J Lab Hematol. 2017; 39(6): 561-568.

15. Semeraro N, Montemurro P, Piccoli C, Muolo V, Colucci M, Giuliani G, Fumarola D, Pece S, Moran AP. Effect of Helicobacter pylori lipopolysaccharide (LPS) and LPS derivatives on the production of tissue factor and plasminogen activator inhibitor type 2 by human blood mononuclear cells. J Infect Dis. 1996; 174(6): 1255-1260.

16. Poli KA, Tofler GH, Larson MG, Evans JC, Sutherland PA, Lipinska I, Mittleman MA, Muller JE, D'Agostino RB, Wilson PW, Levy D. Association of blood pressure with fibrinolytic potential in the Framingham offspring population. Circulation. 2000; 101(3): 264-269.

17. Nordenhem A, Wiman B. Tissue plasminogen activator (tPA) antigen in plasma: correlation with different tPA/inhibitor complexes. Scand $J$ Clin Lab Invest. 1998; 58(6): 475-483.

Received 26.03.2018 